\title{
Separation of Organomercury Species Using Nonaqueous Capillary Electrophoresis Coupled with Sample Stacking and Electrokinetic Injection Techniques
}

\author{
Zhen-Lei Peng', Guanqun Song ', Lixia Zhao', Jin-Ming Lin ${ }^{1,2, \bowtie}$ \\ 1 State Key Laboratory of Environmental Chemistry and Ecotoxicology, Research Center for Eco-Environmental Science, \\ Chinese Academy of Sciences, P.O. Box 2871, Beijing 100085, China; E-Mail: jmlin@mail.rcees.ac.cn \\ 2 Department of Chemistry, Tsinghua University, Beijing 100084, China
}

\begin{abstract}
A nonaqueous capillary electrophoresis (NACE) method, $30 \mathrm{mM}$ ammonium acetate in methanol as background electrolyte (BGE), was developed for separation of the organomercury species without complexing reagents. The effects of different solutes and solvents in BGE were studied. Three species of organomercury, methylmercury, ethylmercury and phenylmercury, were separated well and all the number of theoretical plates were over $10^{6}$. The present NACE method was also coupled with sample stacking and electrokinetic injection techniques to enhance the detection sensitivity. Under the optimum conditions, the limit of detection $(S / N=3)$ is $18 \mathrm{ng} \mathrm{mL}^{-1}$ and the linear relation range from 40 to $750 \mathrm{ng} \mathrm{mL}^{-1}$ were obtained for methylmercury.
\end{abstract}

\section{Keywords}

Capillary electrophoresis

Electrokinetic injection

Sample stacking

Organomercury

\section{Introduction}

There are several kinds of mercury species, elemental, inorganic and organic mercury in nature. The environmental toxicity and biological availability of mercury lie on its chemical form and oxidation state. Among the mercury species, methylmercury $\left(\mathrm{MeHg}^{+}\right)$is the most toxic species, normally found in the environmental and biological samples, because $\mathrm{MeHg}^{+}$can pass through the food chain, accumulating and contaminating humans. Therefore, the total mercury concentration could not reflect the important information about pollutants. Because the concentration of each mercury species is very low in the environmental samples, rapid, sensitive and reliable methods are required for the analysis of mercury species.

Great efforts were made in developing analytical methods for organomercury, such as gas chromatography (GC) [1-5] and high performance liquid chromatography (HPLC) [5-8]. During the recent decade, capillary electrophoresis (CE) has become available as a routine tool for the analysis of environmental and biological samples, especially the speciation of environmental toxic compounds. Many investigations on the analysis of mercury by $\mathrm{CE}$ have been published [5, 9-12]. However, there are some shortcomings to be solved in these CE methods; for example, mercury species need pre-form complexes, which would add the choice procedure of proper ligands and the separation procedure between the complex compounds and excessive ligands. In order to overcome this problem, we developed a nonaqueous capillary electrophoresis (NACE) method [13], acetic acid and imidazole as background electrolyte (BGE), to separate mercury species, which avoided the accession of complexing agents. Due to using the complexing agent, imidazole has high UV adsorption at the detection window of the capillary, which resulted in a low sensitivity for the application. During the last decade, several efficient on-capillary sample concentration methods, e.g., the sample stacking methods, such as sample stacking in aqueous CE [14, 15] and sample stacking in NACE [16-18] have been reported. These methods greatly improved the intensitivity of $\mathrm{CE}$ detection. In the present work, another NACE method without imidazole or other UV adsorption reagents, only with ammonium acetate in methanol as BGE, was developed to separate the mercury species. Combined with electrokinetic injection mode (EI mode) [19-21], a new NACE system was studied for the methylmercury analysis. 
A
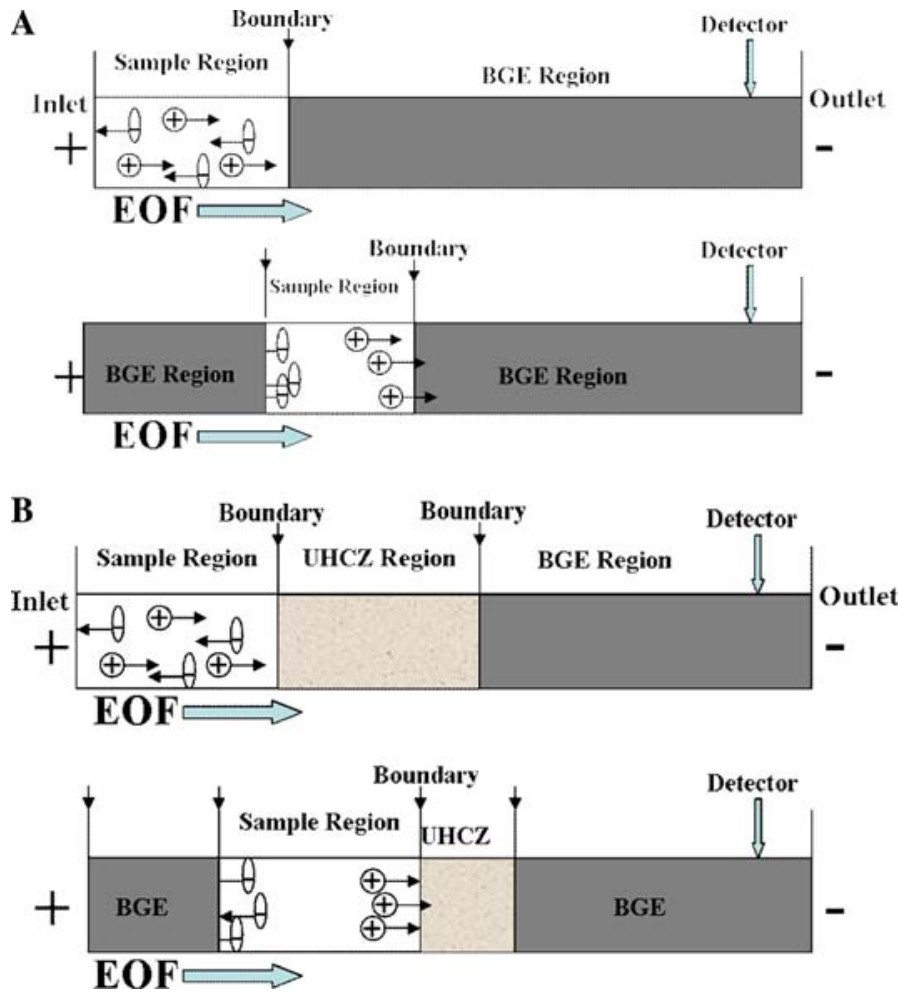

Fig. 1. The schematic diagram of NACE sample stacking. a The large volumes injection of normal NACE sample stacking without the polarity switching; b the large volumes injection of NACEUHCZ-sample stacking

\section{Experimental}

\section{Apparatus}

NACE experiments were performed with a Beckman P/ACE MDQ system (USA), which consists of a $\mathrm{CE}$ unit with diode array detector (DAD) and workstation. Separations were carried out on fusedsilica capillaries of $57 \mathrm{~cm} \mathrm{(50} \mathrm{cm} \mathrm{effective}$ length) $\times 75 \mu \mathrm{m}$ i.d. (Yongnian Optical Fiber Factory, Hebei, China). A detection window was created at $50 \mathrm{~cm}$ from the capillary inlet by removing the polyimide coating. The capillary temperature was maintained at $25^{\circ} \mathrm{C}$ by the cooling system of the CE instrument. The hydrostatic injection mode ( $5 \mathrm{~s}, 0.5 \mathrm{psi}$ ) was used for the injection of the standard sample. Applied voltage was $20 \mathrm{kV}$. Dimethyl sulfoxide (DMSO) was used to mark the electroosmotic flow (EOF). To clean and activate the inner surface, new capillaries were flushed successively for $5 \mathrm{~min}$ with methanol, 5 min with $0.1 \mathrm{M} \mathrm{HCl}, 3 \mathrm{~min}$ with water, 10 min with $0.1 \mathrm{M} \mathrm{NaOH}$, and 5 min with $\mathrm{H}_{2} \mathrm{O}$. Daily before use, the capillary was rinsed for 2 min with $0.1 \mathrm{M}$ $\mathrm{NaOH}, 2$ min with $\mathrm{H}_{2} \mathrm{O}$ and 4 min with running electrolyte. Between analyses, the capillaries were rinsed with running electrolyte at 20 psi for $2 \mathrm{~min}$.

\section{Reagents}

All reagents were of analytical grade or higher. Three different compounds of organic mercuric chloride, methylmercury chloride $\left(\mathrm{MeHg}^{+}\right)$, ethylmercury chloride $\left(\mathrm{EtHg}^{+}\right)$and phenylmercury chloride $\left(\mathrm{PhHg}^{+}\right)$, were purchased from Tokyo Kasei Kogyo Co. (Tokyo, Japan). Ammonium acetate $\left(\mathrm{NH}_{4} \mathrm{Ac}\right)$ and acetic acid (HAc) were produced by Beijing Chemical Company (Beijing, China). Methanol was of chromatographic grade, purchased from Siyou Biology Medical Company (Tianjin, China).

The stock solutions of $\mathrm{MeHg}^{+}, \mathrm{EtHg}^{+}$ and $\mathrm{PhHg}^{+}$were prepared in methanol at concentrations of $250 \mu \mathrm{g} \mathrm{mL}^{-1}$ (as $\mathrm{Hg}$ ), respectively. All stock solutions were protected against light and stored at $4{ }^{\circ} \mathrm{C}$ in the dark. Working standard solutions containing one or more mercury compounds were prepared by stepwise diluting the stock solution to final concentration ( $40 \mathrm{ng} \mathrm{mL}^{-1}$ for each) before use. Warning: these organomercury solutions should be prepared in a well-ventilated hood, and care should be taken to avoid direct contact of these reagents with the skin because of their high toxicity.

The background electrolytes were composed of ammonium acetate with methanol as solvent. Analytical-grade dimethyl sulphoxide (DMSO) was purchased from Sigma (USA). DMSO was used at concentration of $10 \mathrm{mM}$; this solution was prepared either together with the analytes under investigation or directly diluted with the BGEs.

\section{Sample Stacking}

Two different sample stacking methods in NACE were used in this work, normal NACE-sample stacking (see Fig. 1a) and ultra-high conductivity zone NACEsample stacking (UHCZ-NACE-sample stacking) (see Fig. 1b) which is developed by Tsai and his co-workers [18]. In normal NACE-sample stacking, the samples were dissolved in $1 / 100$ diluted $\mathrm{BGE}$ solutions, and large volumes introduced to the capillary. During the separation process, there was no polarity switching. In the UHCZ-NACE-sample stacking mode, an ultra-high conductivity zone was inserted between the sample zone and BGE solution to build a conductivity gradient, the samples were stacked along the capillary axis.

\section{Results and Discussion}

\section{Development of the Normal NACE Method}

In order to find a suitable NACE system for the separation of organomercury species, three different electrolyte systems, ammonium acetate, ammonium formate and sodium acetate, were compared using methanol as organic solvent. In the same operation conditions except for the electrolyte solutes, the running currents were $22.53,24.70$ and $30.83 \mu \mathrm{A}$ for sodium acetate, ammonium acetate and ammonium formate systems, respectively. These results showed that the polarity of ammonium formate was stronger than the others. As shown in Fig. 2, ammonium acetate was more suitable for the separation of organomercury species than the others, which 
might have been caused by its effective mobility matching to that of organomercury; and in addition, the figure showed that the anion played a more important role than the cation for ammonium acetate.

The effect of ammonium acetate concentration on the separation was studied. When the ammonium acetate concentration increased from 20 to $70 \mathrm{mM}$, the detection sensitivity of $\mathrm{MeHg}^{+}$was improved and achieved the maximum value at the concentration of $30 \mathrm{mM}$. The higher concentration of electrolyte solute caused the smaller EOF, which corresponded to a longer migration time. As the electric fields were applied in capillary, the system was tendentious to heat up due to the extra energy, Joule heating. Thereby, the Joule heating generated in the NACE systems was also studied. The running currents had a good linear relationship with the solute concentrations over the tested range, obeying the Ohm's law. It showed that the Joule heating dispersing in these NACE systems were effective, and hence these systems could be available to analyze the organomercury species. These phenomena could also be observed for $\mathrm{EtHg}^{+}$and $\mathrm{PhHg}^{+}$, and considering the general purpose, the amount of $30 \mathrm{mM}$ ammonium acetate was selected as the electrolyte concentration in this work.

When the ammonium acetate concentration and other factors, e.g., the length and diameter of capillary, the applied voltage and temperature were the same, the separations of $\mathrm{MeHg}^{+}, \mathrm{EtHg}^{+}$ and $\mathrm{PhHg}^{+}$were compared with water, methanol, 2-propanol, 1-propanol, acetonitrile, dimethyl sulphoxide, formamide and $N, N$-dimethylformamide as solvents. The results were listed in Table 1. By only using water or methanol as solvent, the three organomercury, $\mathrm{MeHg}^{+}, \mathrm{EtHg}^{+}$ and $\mathrm{PhHg}^{+}$, can be separated and three positive peaks appeared in the chromatogram. No peak or only one peak was recorded when using the other seven solvents. Because water has a higher dielectric constant $(\varepsilon=78.39)$ than that of methanol $(\varepsilon=32.70)$ [19], a larger running current in the water system than in the methanol system was observed, indicating that the Joule heating generated in the water system was larger than that in the methanol system. This is a merit of the NACE systems, which has been well-known.

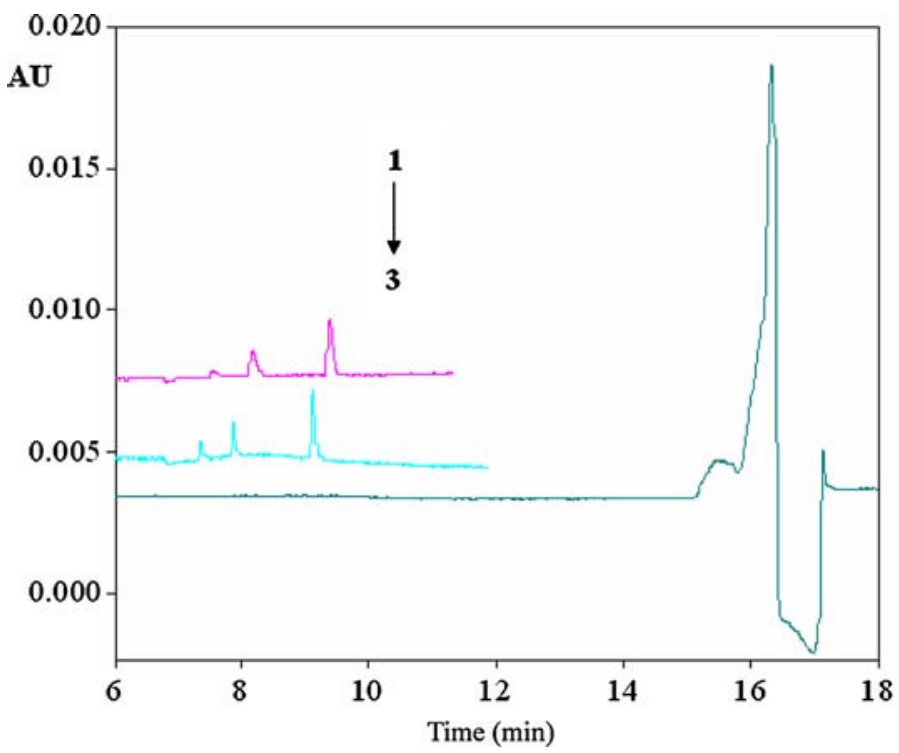

Fig. 2. Electropherograms of three different electrolyte systems for the separation of organic mercury species. $130 \mathrm{mM}$ ammonium formate; $230 \mathrm{mM}$ ammonium acetate; $330 \mathrm{mM}$ sodium acetate. The peaks order were $\mathrm{MeHg}^{+}, \mathrm{EtHg}^{+}, \mathrm{PhHg}^{+}$in turn. Capillary: $57 \mathrm{~cm}(50 \mathrm{~cm}$ effective length) $\times 75 \mu \mathrm{m}$ i.d.; applied voltage: $20 \mathrm{kV}$; temperature: $25^{\circ} \mathrm{C}$; detection wavelength: $191 \mathrm{~nm}$; hydrostatic injection: $0.5 \mathrm{psi} \times 5 \mathrm{~s}$; methanol as solvents

Table 1. The results of different solvents for background electrolytes

\begin{tabular}{|llllllllll|}
\hline & Water & $\mathrm{MeOH}$ & $\mathrm{EtOH}$ & $2-\mathrm{PrOH}$ & 1-PrOH & ACN & DMSO & FA & NMF \\
\hline Current $(\mu \mathrm{A})$ & 41.0 & 26.0 & 4.6 & 0 & 1.0 & 0 & 2.0 & 12.3 & 0.7 \\
Peaks number & 3 & 3 & 1 & 0 & 0 & 1 & 1 & 0 & 1 \\
Peaks Shape & $\mathrm{SP}, \mathrm{PP}$ & $\mathrm{SP}, \mathrm{PP}$ & $\mathrm{NP}$ & - & - & $\mathrm{PP}$ & $\mathrm{NP}$ & $\mathrm{PP}$ & $\mathrm{NP}$ \\
\hline
\end{tabular}

$S P$ Symmetry peak, NP negative peak, $P P$ positive peak, $M e O H$ methanol, Et $O H$ ethanol, 2-PrOH 2-propanol, 1-PrOH 1-propanol, $A C N$ acetonitrile, $D M S O$ dimethyl sulphoxide, $F A$ formamide, $N M F N, N$-dimethylformamide

The repeatability of the migration time, peak area and number of theoretical plates for organomercury species were tested, as listed in Table 2. The relative standard deviations (RSD) were lower than $5.0 \%$, and indicated that this NACE method was suitable for the organic mercury species analysis.

\section{Sample Stacking NACE Method}

Subsequently, in order to get a lower detection limit for the determination of organomercury, the sample stacking methods were studied and applied in the normal NACE system.

The pressure injection time of normal NACE-sample stacking was tested for the most suitable results. When the injection time was increased, the resolutions of organic mercury species decreased (the figure was not shown). Based on that $\mathrm{MeHg}^{+}$was the most toxic species of mercury in the environment, the $\mathrm{MeHg}^{+}$ analysis was considered by normal NACE-sample stacking only. As shown in Table 3, when the injection time increased, the running current decreased to $2.1 \mu \mathrm{A}$. But even when the injection volume of the sample enhanced about 20 -fold from $0.5 \mathrm{psi} \times 5 \mathrm{~s}$ to $0.5 \mathrm{psi} \times$ $99 \mathrm{~s}$, the peak area improvement of methylmercury only changed about 12-fold. When the pressure injection condition was $0.5 \mathrm{psi} \times 99 \mathrm{~s}$, the limit of determination (LOD) was $0.6 \mu \mathrm{g} \mathrm{mL}^{-1}$ $(S / N=3)$, which was not low enough for the determination of methylmercury and still required development of the NACE method.

Based on the UHCZ-NACE-sample stacking method, the UHCZ volumes were tested for the optimum results when the ammonium acetate concentration of UHCZ and other factors, e.g., the electrolyte solution, the injected sample volumes, the length and diameter of the capillary, the applied voltage and temperature were the same. As the 
Table 2. Repeatability of the migration time, peak area and number of the theoretical plates for organic mercury $(n=6)$

\begin{tabular}{|c|c|c|c|c|c|c|c|c|c|}
\hline \multirow[t]{2}{*}{ Times } & \multicolumn{3}{|c|}{ Methylmercury } & \multicolumn{3}{|c|}{ Ethylmercury } & \multicolumn{3}{|c|}{ Phenylmercury } \\
\hline & MT (min) & Area & $N$ & MT (min) & Area & $N$ & MT (min) & Area & $N$ \\
\hline 1 & 7.079 & 1,715 & 111,692 & 7.596 & 3,827 & 69,742 & 8.858 & 3,698 & 149,461 \\
\hline 2 & 7.054 & 1,842 & 110,937 & 7.558 & 3,911 & 68,756 & 8.796 & 3,835 & 160,505 \\
\hline 3 & 7.138 & 1,921 & 109,786 & 7.679 & 4,007 & 64,187 & 8.921 & 3,809 & 152,511 \\
\hline 4 & 7.175 & 1,902 & 102,160 & 7.717 & 4,105 & 66,582 & 8.946 & 3,967 & 160,565 \\
\hline 5 & 7.358 & 1,879 & 101,776 & 7.938 & 4,013 & 65,738 & 9.175 & 3,898 & 145,902 \\
\hline 6 & 7.404 & 1,802 & 111,244 & 7.992 & 3,920 & 70,973 & 9.217 & 3,737 & 146,217 \\
\hline Av & 7.201 & 1,844 & 107,933 & 7.747 & 3,964 & 67,663 & 8.986 & 3,824 & 152,527 \\
\hline RSD \% & 2.03 & 4.13 & 4.32 & 2.31 & 2.46 & 3.82 & 1.91 & 2.61 & 4.36 \\
\hline
\end{tabular}

Conditions: $30 \mathrm{mM} \mathrm{NH} 4 \mathrm{Ac}$ in methanol; temperature: $25^{\circ} \mathrm{C}$; voltage applied: $20 \mathrm{kV}$; capillary: $57 \mathrm{~cm} \times 75 \mu \mathrm{m}(50 \mathrm{~cm}$ effective length) $M T$ Migration time, Area peak area, $N$ number of theoretical plates

Table 3. The normal NACE-sample stacking results for methylmercury $(n=3)$

\begin{tabular}{|c|c|c|c|c|c|c|}
\hline Value $^{\mathrm{a}}$ & $\begin{array}{l}0.5 \mathrm{psi} \\
\times 5 \mathrm{~s}\end{array}$ & $\begin{array}{l}0.5 \mathrm{psi} \\
\times 50 \mathrm{~s}\end{array}$ & $\begin{array}{l}0.5 \mathrm{psi} \\
\times 60 \mathrm{~s}\end{array}$ & $\begin{array}{l}0.5 \mathrm{psi} \\
\times 70 \mathrm{~s}\end{array}$ & $\begin{array}{l}0.5 \mathrm{psi} \\
\times 80 \mathrm{~s}\end{array}$ & $\begin{array}{l}0.5 \mathrm{psi} \\
\times 99 \mathrm{~s}\end{array}$ \\
\hline $\begin{array}{l}\text { Migration } \\
\text { time (min) }\end{array}$ & 7.03 & 6.1 & 5.62 & 5.33 & 4.68 & 4.42 \\
\hline Peak area & 6,867 & 58,495 & 59,238 & 71,660 & 77,770 & 79,533 \\
\hline Current $(\mu \mathrm{A})$ & 25.7 & 4.0 & 3.5 & 2.8 & 2.6 & 2.1 \\
\hline
\end{tabular}

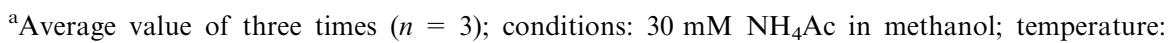
$25^{\circ} \mathrm{C}$; voltage applied: $20 \mathrm{kV}$; capillary: $57 \mathrm{~cm} \times 75 \mu \mathrm{m}(50 \mathrm{~cm}$ effective length)

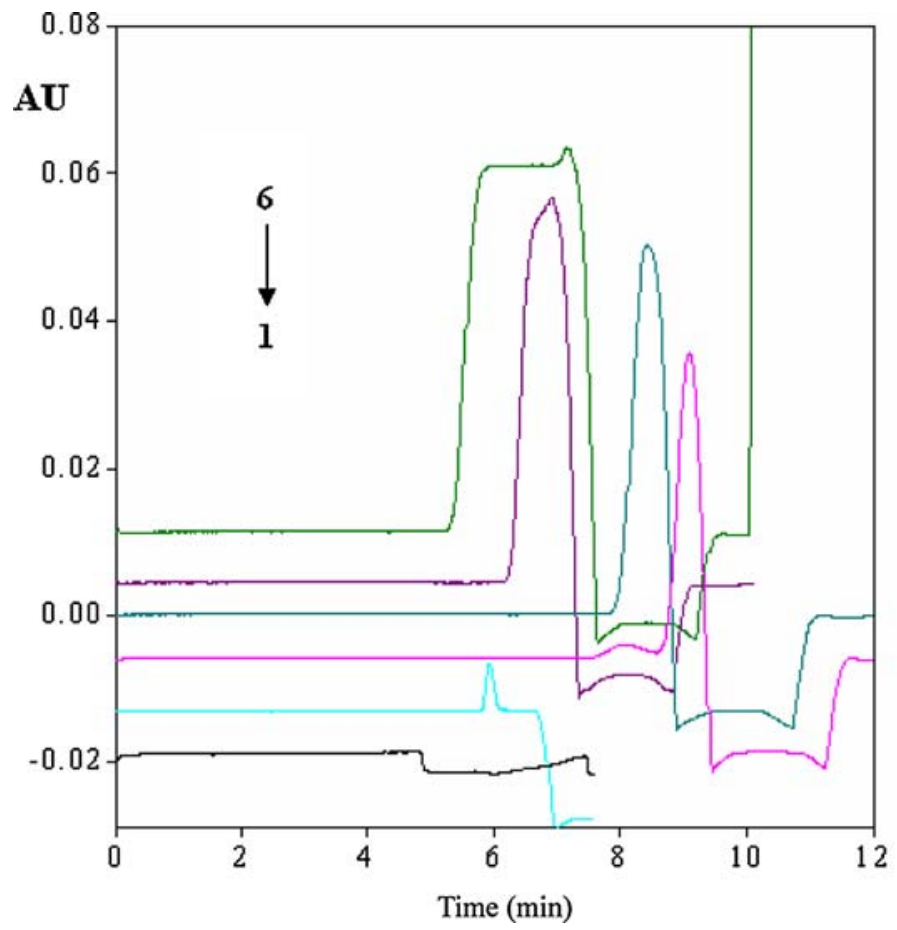

Fig. 3. Electropherograms of the UHCZ volumes effects on the methylmercury analysis. The injection time values of ultra-high conductivity solution from $\mathbf{1}$ to $\mathbf{6}$ were 20,0,5,10,20 and $50 \mathrm{~s}$, respectively as the UHCZ concentration was $200 \mathrm{mM}$. The injection conditions of the sample were all $0.5 \mathrm{psi} \times 50 \mathrm{~s}$ for $\mathbf{2}$ to $\mathbf{6}$; but for $\mathbf{1}$, the injection sample volume was zero; other conditions as in Fig. 2

ammonium acetate concentration of UHCZ was $200 \mathrm{mM}$, the experimental results were illustrated in Fig. 3 which indicated that the UHCZ volumes affected not only the determination of the sensitivity but also the migration time of methylmercury. These results showed that this method was suitable for the improvement of the determination of methylmercury, but only the method was not sensitive enough to determine $\mathrm{MeHg}^{+}$in real samples, and required coupling with other techniques.

As methylmercury has a cation form in methanol, the anode was placed at the inlet side and the cathode at the outlet side of the capillary during the EI mode. Injection time was changed for the enhancement of injection quantity. The results showed that the peak area of methylmercury had a good linear relationship with the injection time at the same applied voltage (the data were not shown).

Even though the sample stacking was obvious by the EI mode, the peak shape of methylmercury was not symmetric and a badly tailing peak appeared. The UHCZ-NACE-sample stacking mode was coupled with the EI mode for improvement of the peak shape and the determination of sensitivity. The concentration and injection volumes of ammonium acetate in the ultra-high conductivity zone were changed for the optimization results. As shown in Fig. 4a, with the increase of the injection time of $300 \mathrm{mM} \mathrm{NH}_{4} \mathrm{Ac}$ into the capillary, the peak area of methylmercury increases to $30 \mathrm{~s}$ before decreasing.

When the injection time was selected at $30 \mathrm{~s}$, the effect of the concentration of ammonium acetate on the detection was studied. The results illustrated in Fig. 4b indicated that the concentration of ammonium acetate at $300 \mathrm{mM}$ was the optimum for the ultra high concentration zone.

\section{The Limit of Detection and Linear Range of Methylmercury}

Under the above optimum conditions, the standard curve for methylmercury was established. The limit of detection 

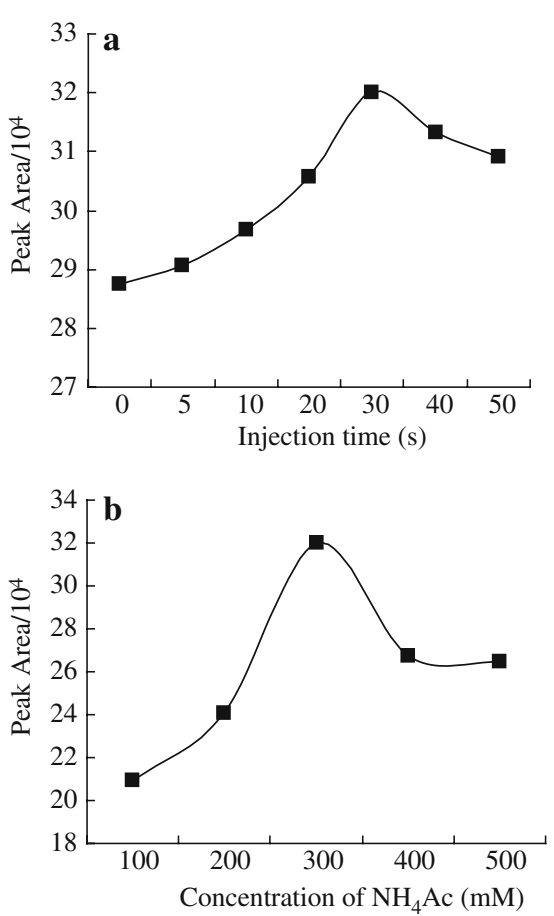

Fig. 4. Effects of the injection time and $\mathrm{NH}_{4} \mathrm{Ac}$ concentration on the determination of methylmercury. The injection conditions: $10 \mathrm{kV} \times 60 \mathrm{~s}$; other conditions as in Fig. 2

of methylmercury was $18 \mathrm{ng} \mathrm{mL}^{-1}$ $(S / N=3)$ which was 33 -fold lower than the normal NACE-sample stacking. The linear relationship between the concentration of methylmercury and peak area was from 40 to $750 \mathrm{ng} \mathrm{mL}^{-1}$ with $191 \mathrm{~nm}$ UV detection. Meanwhile, the applied voltage and injection time of the EI process could be adjusted to enhance the sensitivity and accordingly meet our practical need.

\section{Conclusion}

The NACE method, $30 \mathrm{mM}$ ammonium acetate in methanol as electrolyte, was developed for the separation of organomercury species without complexing reagents. The NACE method, coupled with sample stacking and electrokinetic injection techniques, was developed and applied to enhance the LOD of methylmercury. At the same time, the effect factors on the separation of organomercury were tested and discussed for the optimum results. The electrolytes were stable and very easy to prepare, avoiding problems associated with adjusting $\mathrm{pH}$ and $\mathrm{pH}$ error between the experiments.

\section{Acknowledgments}

This work was supported by the National Natural Science Foundation of China (Nos. 20437020, 20575008) and the Major Research Program of Chinese Academy of Sciences (KZCX3-SW-432).

\section{References}

1. Dietz C, Madrid Y, Camara C, Quevauviller P (2000) Anal Chem 72:4178-4184
2. Munoz J, Gallego M, Valcarcel M (2004) J Chromatogr A 1055:185-190

3. Gomez-Ariza JL, Lorenzo F, Garcia-Barrera T (2005) Appl Organometal Chem 19:600-604

4. Pereiro IR, Diaz AC (2002) Anal Bioanal Chem 372:74-90

5. Carro AM, Mejuto MC (2000) J Chromatogr A 882:283-307

6. Bramanti E, Lomonte C, Onor M, Zamboni R, D'Ulivo A, Raspi G (2005) Talanta 66:762-768

7. Palenzuela B, Manganiello L, Rios A, Valcarcel M (2004) Anal Chim Acta 511:289-294

8. Qvarnstrom J, Tu Q, Frech W, Ludke C (2000) Analyst 125:1193-1197

9. Pager Cs, Gaspar A, (2002) Microchem J 73:53-58

10. Lee TH, Jiang SJ (2000) Anal Chim Acta 413:197-205

11. Rocha MS, Soldado AB, Blanco-González E, Sanz-Medel A (2000) J Anal At Spectrom 15:513-518

12. Manganiello L, Arce L, Rios A, Valcarcel M (2002) J Sep Sci 25:319-327

13. Peng ZL, Qu F, Song G, Lin JM (2005) Electrophoresis 26:3333-3340

14. Quirino JP, Terabe S (2000) J Chromatogr A 902:119-135

15. Carro-Díaz AM, Lorenzo-Ferreira RA, Cela-Torrijosl R (1996) J Chromatogr A 730:345-351

16. Tsai CH, Fang C, Liu JT, Lin CH (2004) Electrophoresis 25:1601-1606

17. Jang J, Koo YM, Chung DS (2003) J Capillary Electrophor (US) 8:19-24

18. Tsai CH, Tsai CC, Liu JT, Lin CH (2005) J Chromatogr A 1068:115-121

19. Chien RL, Burgi DS (1991) J Chromatogr 559:141-152

20. Zhang CX, Thormann W (1996) Anal Chem 68:2523-2532

21. Chien RL (1991) Anal Chem 63:2866-2869 